\title{
The structure of liquid alkali nitrates and nitrites.
}

\author{
Martin C. Wilding ${ }^{1}$, Mark Wilson ${ }^{2}$, Mauro C. C. Ribeiro $^{3}$, \\ Chris J. Benmore ${ }^{4}$, J.K.R. Weber ${ }^{4,5}$, Oliver Alderman ${ }^{4,5}$, \\ Anthony Tamalonis ${ }^{5}$ and J. B. Parise ${ }^{6}$. \\ 1 Department of Chemistry, University College London, 20 Gordon Street, London, \\ WC1H 0AJ, UK \\ 2 Department of Chemistry, Physical and Theoretical Chemistry Laboratory, \\ University of Oxford, South Parks Road, Oxford OX1 3QZ, UK \\ ${ }^{3}$ Laboratório de Espectroscopia Molecular, Instituto de Química, Universidade de \\ Sāo Paulo, Av. Prof. Lineu Prestes 748, Sāo Paulo, 05508-000, Brazil. \\ 4 X-ray Science Division, Argonne National Laboratory, Argonne, IL60439, U.S.A. \\ 5 Materials Development Inc, Arlington Heights, IL60004, U.S.A. \\ ${ }^{6}$ SUNY, Stony Brook, NY U.S.A.
}




\begin{abstract}
.
High energy X-ray diffraction has been combined with containerless techniques to determine the structure of a series of alkali and ammonium nitrate and nitrite liquids. The systems have been modelled using molecular dynamics simulation which allows for the flexibility of, and movement of charge within, the molecular anions. The model reproduces the experimentally-determined scattering functions in both the low- and high $-Q$ regimes reflecting the inter- and intra-molecular length-scales. For ammonium nitrate the best fit to the diffraction data is obtained by assuming the $\mathrm{NH}_{4}^{+}$cation to have a radius closer to that for $\mathrm{Cs}^{+}$rather than a smaller cation such as $\mathrm{Rb}^{+}$as often previously assumed. The alkali nitrites show an emergent length scale, attributed to the nitrogen-nitrogen spatial correlations, that depends on both temperature and the identity of the alkali cation. The corresponding nitrates show a more subtle effect in the nitrogen-nitrogen correlations. As a result, the nature of this N-N length-scale appears different for the respective nitrites and nitrates.
\end{abstract}

\title{
1. Introduction.
}

Molten salts are employed in a range of different applications which have made them the subject of a large number of detailed studies over several decades [1, 2, 3, 4]. Molten salts generally have properties which make them particularly useful in energy applications. They can dissolve actinides and can be used in moderation of nuclear reactions, accordingly they were studied extensively in the 1950s and 1960s for potential use in molten salt reactors $[5,6,7,8]$. Molten salts are very effective as heat transfer fluids and do not require pressurising. These advantages have led to non-nuclear applications with two important nitrate-based salts; solar salt $\mathrm{NaNO}_{3}-\mathrm{KNO}_{3}$ and Hitec ${ }^{\mathrm{TM}}$ [9] $\mathrm{NaNO}_{3}$ $\mathrm{NaNO}_{2}-\mathrm{KNO}_{3}$ being used in renewable energy applications [10, 11, 12, 13].

Comprehensive databases were developed by Janz [14, 15] and summarized in 1988 [14], providing detail on density, viscosity, surface tension and electrical conductivity. The molten salt databases all suggest that the liquids have low viscosity with an Arrhenius viscosity-temperature relation and that simple, linear molar addition can be used to model liquid mixtures and generally suggest a relatively uncomplicated liquid behaviour. However, recent studies suggest that the liquid structure may be more complicated than that previously considered and that the liquid viscosity may be influenced by development of low-dimensional networks $[16,17]$.

The simplest view of molten salt structure is to assume molecular anions, such as sulphate $\mathrm{SO}_{4}^{2-}$, carbonate $\mathrm{CO}_{3}^{2-}$, nitrate $\mathrm{NO}_{3}^{-}$or nitrite $\mathrm{NO}_{2}^{-}$, with a fixed molecular geometry and charge distributions which are then surrounded by cations (e.g. alkalis or $\mathrm{NH}_{4}^{+}$) $[18,19]$. However, this model does not fully explain experimentally-observed properties such as self-diffusion coefficients [20] indicating that more chemically-realistic liquid models are required $[21,22,23,24]$. Evidence is emerging that systems previously considered structurally "simple" (meaning classically ionic with fixed geometries and charge distributions) may show more complex and highly temperature-dependent structure which may have significant implications for the dynamics, viscosities and 
related fragilities [25]. A generic feature of fragile liquids which is often overlooked is that they show Arrhenius behaviour at high temperatures, typically with lower viscosities when compared to stronger liquids, and show departure from this behaviour on supercooling. As a result, whilst these molten salts may be expected to show low viscosities at high temperatures (consistent with the databases of Janz [14, 15, 26]) the supercooled liquids will be more likely to show increases in viscosity that may vary with system (i.e. these will be fragile liquids). Understanding properties such as viscosity (and their relationship to the detailed atomistic structure) requires effective modelling of liquid structures over a range of temperatures.

The determination of liquid structure at high temperature is not trivial since sample containers and furnaces contribute to the total scattered signal. The development of containerless techniques $[27,28,29,30,31]$, which can be combined with X-ray or neutron diffraction $[32,29,30,31]$ means that the study of many types of liquids is now feasible, including metallic systems [33, 34], refractory oxides [35, 36, 37] and molten salts [17]. The liquid structure can be interpreted by reverse Monte Carlo or empirical potential (EPSR) models, techniques which fit an atomic configuration to the liquid diffraction data [38]. However, these do not provide a detailed understanding of the atomic scale interactions and, furthermore, do not provide a means for exploring additional parameter space. Molecular dynamics simulations offer the opportunity to study the liquid state by developing realistic inter-atomic potentials based on the physical chemistry of the liquid components and can be used to explore the temperatureand composition-dependence of liquid properties and extract additional information such as the diffusivities of component ions. The parallel development of state-of-theart diffraction techniques that combine high energy X-ray diffraction (HEXRD) with containerless processing and similarly-advanced molecular dynamics simulations offers an opportunity to study the structure and related properties of molten salts. In this contribution we will combine these approaches to investigate the properties of molten nitrates and nitrites.

The development of potential models which incorporate the effects of polarisation have proved significant in successful modelling of molten salts $[39,40]$. For ionic systems, with ions generated from single atoms, polarization may take the form of moments arising as a response of the electron density to an internal field (or gradient) [41, 39]. The magnitudes of these responses are controlled by the ion polarizabilities (which may be obtained by reference to experiment or electronic structure calculations). For molecular ions the charge distribution of the constituent atoms may be varied, for example, in a fluctuating charge model (FCM) $[42,43,44,45]$ subject to constraining the total charge to being the (formal) molecular ion charge. The different magnitudes of the charges on the atoms are controlled by the respective atomic electronegativies [42]. Fluctuating charge models of this type may employ a rigid description of the molecular geometry (see, for example, refs. $[42,43,44,45,46,44,47,48,49])$ or allow the geometry to be flexible (see, for example, ref. [50]). For planar molecules such as $\mathrm{NO}_{3}^{-}$(and, obviously, $\mathrm{NO}_{2}^{-}$) a rigid FCM will not facilitate any polarization out of the molecular plane. A flexible 
description clearly allows out of plane polarization. However, care may be required in developing these models as the use of both fluctuating charges and a flexible geometry allows out of plane polarization to be generated both from changes in the geometry and/or the charge distribution.

In a recent study we combined containerless processing techniques, HEXRD and molecular dynamics simulation to study the structure and dynamics of molten sodium carbonate [17]. Earlier studies of molten carbonates [51] combined diffraction data with simple ionic models assuming rigid carbonate anions. The more recent [17] study suggests that the underlying liquid structures are more complex and strongly temperature dependent. The total structure factor, $F(Q)$, for molten $\mathrm{Na}_{2} \mathrm{CO}_{3}$ was determined by HEXRD at temperatures of $T=1255-1328 \mathrm{~K}$ for a range of levitation gases and compared with the results of a much earlier diffraction study of Zarzycki [51]. Although there is good agreement with the positions of the main peaks in the $F(Q)$ of the earlier study the recent HEXRD data shows differences in intensity and resolution and accesses a much higher $Q$-range. Simulation models, employing a flexible description of the molecular anion, reproduce the total structure factors and allow the breakdown into the underlying partial structure factors, as well as any temperature-dependence of structure, to be investigated. At high $Q$ the oscillations in the $F(Q)$ represent short range order and represent the superposition of the $\mathrm{C}-\mathrm{O}$ and $\mathrm{O}-\mathrm{O}$ partial contributions, with intensities of these oscillations determined by the rigidity of the anion. The simulations using the flexible anion match both high- and low- $Q$ parts of the $F(Q)$, with differences in intensity of the low- $Q$ peaks attributed to the temperature-dependence of the structure[17]. Analysis in real space shows changes in the C-C correlations and indicates the formation of a chain-like network of carbonate anions, the lengths of which decrease as the temperature increases. The diffusivities show that the sodium ions move considerably more rapidly when compared to those observed for silicates and are clearly correlated with the chain length. The structure of the carbonate liquid reflects the formation of transient, low-dimensional networks which strongly influences diffusion and correlates with the changes in viscosity (and configurational entropy) when the liquids are deeply supercooled.

In this Paper we extend this methodology to explore the structures of pure nitrate and nitrite liquids. Some of these liquids have previously been studied using neutron and/or X-ray diffraction $[21,22,23,24,52,53,54]$ and by molecular dynamics simulation $[49,48,47,46,50]$. These two liquid types have different molecular anions; nitrate $\left(\mathrm{NO}_{3}^{-}\right)$and nitrite $\left(\mathrm{NO}_{2}^{-}\right)$. The key goal of this study is to develop realistic models for both nitrate and nitrite liquids based on a single model for the two anions and which will be compared with high energy X-ray diffraction data. The use of a single model for the two anions will better facilitate future study of the mixtures as well as exploring the temperature-dependence of key structural properties. Here we study the end-member alkali nitrates, $\mathrm{MNO}_{3}(\mathrm{M}=\mathrm{Li}, \mathrm{Na}, \mathrm{K})$ and nitrites, $\mathrm{MNO}_{2}(\mathrm{M}=\mathrm{Na}, \mathrm{K})$ as well as $\mathrm{NH}_{4} \mathrm{NO}_{3}$, well-known as one of the main ingredients in commercial explosives. Although this latter nitrate is challenging to handle experimentally (it will decompose 
into $2 \mathrm{H}_{2} \mathrm{O}+\left[\mathrm{N}_{2} \mathrm{O}\right.$ or $\left.\mathrm{N}_{2}+\frac{1}{2} \mathrm{O}_{2}\right]$ and can be induced to decompose explosively) it is often classed as an effective alkali nitrate, with the conventional view that the $\mathrm{NH}_{4}^{+}$ion has an ionic radius similar to $\mathrm{K}^{+}$or $\mathrm{Rb}^{+}[55]$. Finally, we will study $\mathrm{LiNO}_{2}$ using simulation only in order to present a full set of nitrites corresponding to the nitrates considered.

\section{Experimental Background.}

\subsection{Materials.}

The samples for levitation were synthesised by melting nitrate and nitrite reagents (Sigma Aldrich 99.99\%). The sample masses were of the order of 50-100mg. The samples were fused on a water-cooled copper hearth and heated by a $100 \mathrm{~W} \mathrm{CO}_{2}$ laser to form $\sim 3-4 \mathrm{~mm}$ beads. Sample contamination has been shown to be negligible when this method is used.

In this study we have determined the liquid structures of the nitrates $\mathrm{NH}_{4} \mathrm{NO}_{3}$, $\mathrm{LiNO}_{3}, \mathrm{NaNO}_{3}$ and $\mathrm{KNO}_{3}$ and the nitrites $\mathrm{KNO}_{2}$ and $\mathrm{NaNO}_{2}$. The change in mass for

each sampled was calculated by determining the mass before and after each diffraction measurement and was found to be $\lesssim 2 \%$. The ammonium nitrate liquid was the exception and decomposed when heated on a time-scale of 30-60 seconds limiting the duration of the diffraction measurement. Decomposition to $\mathrm{N}_{2} \mathrm{O}$ (or $\mathrm{N}_{2}$ and $\mathrm{O}_{2}$ ) and $\mathrm{H}_{2} \mathrm{O}$ was indicated by a loss of most of the sample mass and "smoke" produced on heating. Lithium nitrate showed a slight gain in mass (although still within $2 \%$ ) following the levitation experiments, most likely due to limited hydration. X-ray diffraction data was collected on recovered beads (using a laboratory diffractometer) to determine whether the compositions had changed during the process of levitation. The resulting diffraction patterns confirm the partial hydration of the Li and K-nitrate samples following the HEXRD experiments.

\subsection{Containerless Methodology.}

Containerless techniques can be combined with laser heating and high energy Xray diffraction to obtain liquid diffraction patterns. There are many advantages of the containerless techniques including the absence of a need to remove background contributions from a sample holder from any diffraction measurement, the lack of potential reaction between the liquid and container and subsequent contamination and also, in the absence of heterogeneous nucleation sites, the opportunity to observe deeply supercooled liquids [30, 29, 56].

The conical nozzle levitator (CNL) is a containerless sample environment that can be fully integrated into the high energy beam line 6-ID-D at the Advanced Photon Source (APS), Argonne National Laboratory. The nitrate and nitrite sample beads are placed in a purpose-designed, water-cooled conical nozzle, levitated by a gas flowing through the nozzle and heated by a $400 \mathrm{~W}$ continuous wave $\mathrm{CO}_{2}$ laser. The entire levitator is enclosed in a water-cooled stainless steel chamber. Lateral sample ports allow operation 
in transmission mode. The inlet and outlet ports have Kapton windows. Additional, angled ports allow the sample to be viewed by a video camera and pyrometer.

The sample temperature was measured by an optical pyrometer (wavelength $5 \mu \mathrm{m}$ and which covers a temperature range of $T=500-900 \mathrm{~K}$ ) that views the samples though one of the angled ports. The apparent temperatures were corrected using Wien's displacement law and a spectral emissivity of 0.96 [57]. Table 1 summarises key temperature data for the six systems investigated here by HEXRD. The table lists the sample temperatures (obtained from the pyrometer) along with the published melting points and decomposition temperatures [58, 59]. In all cases the decomposition temperatures represent the onset of chemical change which may be kinetically hindered enough to allow HEXRD patterns to be obtained for the (metastable) liquid. Decomposition experiments carried out under different conditions may generate differing results as the removal (or not) of the gas generated from the decomposition process may affect the decomposition pathway.

Earlier measurements made on sodium carbonate [17] informed the methodology used in this contribution, a more diffuse, de-focussed laser beam was used to heat the sample which, when combined with larger drop sizes meant that the liquid levitated more stably without oscillations. The nitrate and nitrite samples are levitated by Ar:5 vol $\% \mathrm{CO}_{2}$ gas.

\subsection{High energy X-ray diffraction}

Diffraction measurements were made at beam line 6-ID-D at the Advanced Photon Source (APS), Argonne National Laboratory. The incident X-rays had an energy of $100.329 \mathrm{keV}(\lambda=0.12369 \AA)$. The beam was collimated to $0.2 \mathrm{~mm} \times 0.5 \mathrm{~mm}$. In addition to high energies the first super-conducting undulator (SCU0) was installed at sector 6 in 2013 [60, 61] which has resulted in an approximate 10-fold increase in photon flux (at $100 \mathrm{keV}$ ) when compared to other insertion devices.

This small incident X-ray beam and high brightness mean that data can be collected in seconds or even more rapidly. This offers the opportunity to evaluate the changing structure that is suggested in fragile liquids. It also allows for rapid measurements on melts prior to decomposition or evaporation.

The 2D liquid diffraction pattern is obtained from a vertically mounted Tl-doped CsI scintillation detector (Perkin Elmer, 1621 AN3) comprising 2048 x 2048 pixels. The sample-detector distance $(353.0691 \mathrm{~mm})$ and tilt angle of the detector are determined by refining to a polycrystalline $\mathrm{CeO}_{2}$ standard. Each measurement of the stable liquid comprised a summed series of 120 frames, each of 0.1 s exposure. The diffraction patterns were reduced from 2D images and corrected for the effects of polarization and geometry using the Fit2D program [62]. A separate measurement of the sample background was made using the same exposure times with no sample in the nozzle but with levitation gas still flowing. The structure factors were obtained using the program PDFget X2 [63] which removes the sample background and corrects for sample absorption, geometric 
and detector-related effects [64].

\subsection{Simulation Methods.}

The methodology chosen here is to employ a flexible description of the molecular ion geometry coupled with a fluctuating charge model (in which the atomic charge distributions on each molecular ion may fluctuate). This builds on our previous work (focussed on $\mathrm{Na}_{2} \mathrm{CO}_{3}$ [17]) in which a flexible molecular ion description was employed but with a fixed charge distribution. Our key aim in introducing the FCM is to attempt to consistently model both the nitrates and nitrites with the same basic model.

2.4.1. Model Basis. In many ways the key to modelling molecular ions is to understand (and effectively replicate) the intramolecular charge distribution and its response to the presence of other ions (fields). At the simplest level (partial) charges may be placed on the atomic centres whose sign and magnitude reflects some known molecular property. A classic textbook starting point for the charge distributions is to consider the resonance structures [55]. A resonance consideration for the nitrite and nitrate anions would predict charges of $\left\{q_{O}, q_{N}\right\}=\left\{-\frac{1}{2}, 0\right\} e$ and $\left\{-\frac{2}{3},+1\right\}$ e respectively. These charges do not, however, reflect those obtained by more detailed analysis. Previous models have employed oxygen atom charges in the range $q_{O}=-0.5533 e$ to $-0.766 e$ for the $\mathrm{NO}_{3}^{-}$ion $[65,66,67,68,69,70,52,71,72]$ and $q_{O}=-0.41 e$ to $-0.75 e$ for the $\mathrm{NO}_{2}^{-}$ $[71,73,74,75,76,77,78]$ obtained with respect to experiment and ab initio electronic structure calculations.

2.4.2. The Fluctuating Charge Model. The longer-term aim of effectively modelling mixtures of nitrates and nitrites (and, beyond that, mixtures containing different molecular anions) dictates that a single model which controls the charge distribution on both molecular anions is preferable to mixing two separate models. In a FCM the molecular charge distribution is controlled through the electronegativities of the constituent atoms (which are dependent upon the atom environment) [42, 45, 44]. The electronegativity on a given site $\alpha$ on ion $i$ is given by

$$
\chi_{i \alpha}=\chi_{i \alpha}^{0}+J_{\alpha \alpha}^{0} q_{i \alpha}+\sum_{\beta \neq \alpha} J_{\alpha \beta} q_{i \beta}+\sum_{j \neq i} \sum_{\beta} \frac{q_{j \beta}}{r},
$$

where $\chi_{i \alpha}^{0}, J_{\alpha \alpha}^{0}$ and $J_{\alpha \beta}$ are parameters. $J_{\alpha \alpha}^{0}$ and $J_{\alpha \beta}$ effectively control the charge balance between different sites on the same molecular ion, whilst the other ions in the system affect the charge distribution on the molecular ion via the final (coulombic) interaction term. Parameters may be obtained by fitting to experimental properties or by reference to ab initio calculations (see, for example, refs. [43, 79]). Significant previous work has focussed on the key fragile glass-former CKN [49, 46, 48] as well as the alkali nitrates $[47,50]$. 
2.4.3. Model Details. Simulations are performed using the FCM on systems containing 512 molecules in the NVT ensemble over a temperature range of $T=600-1000 \mathrm{~K}$ with the temperature controlled using Nosé-Hoover thermostats [80, 81]. The system densities are taken from standard sources for the alkali systems $[14,15]$ and for $\mathrm{NH}_{4} \mathrm{NO}_{3}$ from $[82]$.

The equations governing the time-evolution of the fluctuating charges are integrated in parallel to the Newtonian equations governing the ion motion using a Car-Parrinello method [83] as described in ref. [44]. The potential models are based on those used previously [47]. The ion-ion short-range interactions are accounted for by Born-Mayer potentials and dipole-dipole dispersive interactions (as described in ref. [47]). In the majority of previous models the molecular anion has been fixed although a flexible description has been developed [50]. Here we adapt our previous method of constraining the anion geometry by joining the $\mathrm{N}-\mathrm{O}$ and $\mathrm{O}-\mathrm{O}$ pairs with harmonic springs $[84,17]$ and over-laying a FCM. The harmonic force constants are taken from those used previously for $\mathrm{Na}_{2} \mathrm{CO}_{3}$ [17] with $\mathrm{N}-\mathrm{O}$ and $\mathrm{O}-\mathrm{O}$ equilibrium spring lengths of $1.19 \AA$ and $2.26 \AA$ respectively. The FCM parameters are taken from reference [47]. The inclusion of both variation of the internal charge distribution and bond flexibility introduces two mechanisms by which moments may vary. For example, a dipole may be generated by increasing the charge separation or by lengthening a bond (with fixed charges). We note, however, that the model reflects the situation in a real molecule.

\section{Results.}

In considering the system structures we repeat our previous strategy [17] of surveying both the experimental and simulation results first in reciprocal space before then focussing on the simulation results to obtained detailed information in real space. The choice of this strategy reflects the fact that the experimental data is generated in reciprocal space and that analogous simulation information can be obtained directly from the atom positions. The latter is not entirely trivial since the total scattering functions (which are what are measured experimentally) must be then constructed from the underlying partial structure factors and, as a result, the respective scattering powers ( $Q$-dependent form factors) of the constituent atoms must be known. Since the experimental information is available at a total structure factor level only then the Fourier transforms will reflect the superposition of the six underlying partial pair distribution functions (ten for $\mathrm{NH}_{4} \mathrm{NO}_{3}$ ). In systems of this type, however, the high $-Q$ regime would be expected to be dominated by the well-defined molecular anion geometry and, as a result, extracting reliable structural information on other length-scales may be problematic. Reliable simulation models may uncover detailed structural information effectively buried in the experimentally-determined total scattering functions.

The total X-ray scattering function, $F^{x}(Q)$, is generated by combining the partial (Ashcroft-Langreth) structure factors (of which there are six for the three component system). These are calculated directly from the Fourier components of the ion densities, 
$S_{\alpha \beta}(Q)=\left\langle A_{\alpha}^{*}(Q) A_{\beta}(Q)\right\rangle$, where $A_{\alpha}(Q)=\frac{1}{\sqrt{N_{\alpha}}} \sum_{j=1}^{N_{\alpha}} \exp \left(i \mathbf{Q} \cdot \mathbf{r}^{j}\right)$. Total X-ray structure factors are constructed from weighted sums of these partial structure factors

$$
F^{x}(Q)=\sum_{\alpha} \sum_{\beta} c_{\alpha}{ }^{1 / 2} c_{\beta}{ }^{1 / 2} f_{\alpha}(Q) f_{\beta}(Q)\left[S_{\alpha \beta}(Q)-\delta_{\alpha \beta}\right]
$$

where $c_{\alpha(\beta)}$ is the mole fraction of species $\alpha(\beta)$ and $f_{\alpha(\beta)}(Q)$ are the X-ray form factors and are taken from standard sources $[85,86]$.

\subsection{Nitrates. Q-space.}

3.1.1. $\{\mathrm{Li}, \mathrm{Na}, \mathrm{K}\} \mathrm{NO}_{3}$. Figure 1 shows the experimental total scattering functions, $F(Q)$, for four pure nitrates and two pure nitrites as indicated. The relative X-ray weightings of the contributing atoms mean that the nitrate anions will dominate the $\mathrm{LiNO}_{3}$ and $\mathrm{NH}_{4} \mathrm{NO}_{3}$ functions whilst the $\mathrm{K}^{+}$cation will dominate both the $\mathrm{KNO}_{2}$ and $\mathrm{KNO}_{3}$ functions. The sodium salts would be expected to show a relatively even balance of contributions from the underlying partial structure factors. The well-defined geometries of the respective molecular anions should dominate the high $-Q$ oscillations [87]. Figure 1 also shows the experimental total scattering functions, $F(Q)$, for the same six systems as $Q(F(Q)-1)$ in order to emphasize any oscillations at high $Q$ which, as expected, show in-phase oscillations for each molecular anion.

Figure 2(a) shows the total scattering function obtained from simulation for $\mathrm{LiNO}_{3}$ (determined at $T=800 \mathrm{~K}$ ) compared to that obtained from HEXRD experiments and the breakdown into the X-ray weighted partial structure factor contributions. The agreement between the simulated and experimental $F(Q)$ functions appears excellent across the entire $Q$-range. The relative scattering power of both the $\mathrm{N}$ and $\mathrm{O}$ atoms compared with $\mathrm{Li}$ (and the number of $\mathrm{O}$ atoms in the formula unit) means that $F(Q)$ is dominated by the contributions from $S_{O O}(Q)$ and $S_{N O}(Q)$. As a result $F(Q)$ for this system very much reflects the structure and distribution of the molecular anions. To aid this comparison figure 2(a) also shows the respective functions as $Q(F(Q)-1)$ and $Q\left[S_{\alpha \beta}(Q)-\delta_{\alpha \beta}\right]$ in order to highlight the high $Q$ oscillations which are clearly arising from out of phase oscillations in the NO and OO correlations.

Figure 2(b) shows the breakdown of $F(Q)$ (determined at $T=800 \mathrm{~K}$ ) for $\mathrm{NaNO}_{3}$ into the weighted partial structure factor contributions with figure 2(b) showing $Q(F(Q)-1)$ and $Q\left[S_{\alpha \beta}(Q)-\delta_{\alpha \beta}\right]$. The constituent atoms are now of approximately equal scattering power and so the overall $F(Q)$ is a subtle superposition of significant contributions from each partial structure factor. Figure 2(c) shows the breakdown of $F(Q)$ (determined at $T=800 \mathrm{~K}$ ) for $\mathrm{KNO}_{3}$ into the weighted partial structure factor contributions with figure $2(\mathrm{c})$ showing $Q(F(Q)-1)$ and $Q\left[S_{\alpha \beta}(Q)-\delta_{\alpha \beta}\right]$. The scattering power of the $\mathrm{K}^{+}$ions dominate $F(Q)$. The agreement is not as impressive as for the Li or $\mathrm{Na}$ analogues but not bad nevertheless. The implication may be that the alkali-oxygen term in the pair potential may transfer less well between alkalis than some of the other terms. This potential problem is more stark for the potassium salt given the relatively large scattering power of the $\mathrm{K}^{+}$cation. 
3.1.2. $\mathrm{NH}_{4} \mathrm{NO}_{3}$. A useful starting point for considering $\mathrm{NH}_{4} \mathrm{NO}_{3}$ is to assume the cation to be spherical with a radius matching that for $\mathrm{K}^{+}$or $\mathrm{Rb}^{+}$as discussed in the introduction [55]. Figure 3 shows the comparison of the total scattering functions obtained from HEXRD experiment and from the model for $\mathrm{KNO}_{3}$ using the respective $\mathrm{NH}_{4} \mathrm{NO}_{3}$ form factors (assuming the $\mathrm{H}$ atoms to be X-ray invisible). Figure 3 also shows the breakdown into the X-ray weighted partial structure factor contributions. The high $-Q$ oscillations again reflect the geometry of the $\mathrm{NO}_{3}^{-}$anion and so are well reproduced. The low $-Q$ features (at $Q<3 \AA^{-1}$ ) are less well accounted for. To assess whether these differences simply reflect the difference between the $\mathrm{K}^{+}$cation radius and the effective radius for the $\mathrm{NH}_{4}^{+}$cation, the cation size is systematically increased. Reproducing the experimental total scattering function represents a stern test of the model as the constituent atoms (here $\mathrm{N}$ (twice) and $\mathrm{O}$ ) have very similar X-ray scattering powers and so all six partial structure factors contribute significantly to the total scattering function. Figure 3 shows the effect of increasing the cation radius (retaining all other terms in the potential model) by $\Delta \sigma=0.25 \AA$ on the total scattering function whilst figure 3 shows the respective breakdown into the partial structure factors. The increased cation size significantly improves the agreement with the experimental total scattering function, in particular in the relatively low $-Q$ regime. The required scaling of $\Delta \sigma=0.25 \AA$ is larger than one would expect if the $\mathrm{NH}_{4}^{+}$cation were behaving as $\mathrm{Rb}^{+}$, the respective Shannon radii of $\mathrm{K}^{+}$and $\mathrm{Rb}^{+}$being $1.38 \AA$ and $1.49 \AA$ (VIcoordinate) respectively[88, 89]. Furthermore, although Shannon deliberately omitted $\mathrm{NH}_{4}^{+}$from his original consideration[88], recent work predicts a cation radius of $1.48 \AA$ [90]. In the present work, therefore, a change in cation radius which places $\mathrm{NH}_{4}^{+}$nearer to $\mathrm{Cs}^{+}$(radius $1.70 \AA$ ) is required.

There are a number of potential explanations as to why a seemingly relatively large cation is required. For example, the ammonium ion may be harder or softer than the typical alkali cations. In the simulation models employed here the ion "hardness" is controlled by a single parameter presumed fixed on transferring between different cations. It is possible that the approximation of fixing this parameter for the $\mathrm{NH}_{4}^{+}$ cation in inappropriate. Alternatively (although potentially related to the previous point) the molecular nature of the $\mathrm{NH}_{4}^{+}$cation itself may be significant and require explicit inclusion in terms of imposing both a tetrahedral local geometry and a suitable charge distribution. The detailed origin of this difference will be the subject of a future investigation. For the moment, however, it is sufficient to note that the total scattering function for molten $\mathrm{NH}_{4} \mathrm{NO}_{3}$ can be reproduced using the same class of potentials which describe the alkali metal systems. Furthermore, the use of a relatively large ion size is consistent with results obtained from neutron scattering experiments [22].

\subsection{Nitrites. Q-space.}

Figure 4(a) shows the breakdown of $F(Q)$ (determined at $T=800 \mathrm{~K}$ ) for $\mathrm{LiNO}_{2}$ into the X-ray weighted partial structure factors. No experimental data is yet available 
for this system but it is clear that, as would be expected, $F(Q)$ is dominated by the contributions from the $\mathrm{NO}$ and $\mathrm{OO}$ pairs. Figure $4(\mathrm{~b})$ shows the breakdown of $F(Q)$ (determined at $T=800 \mathrm{~K}$ ) into the weighted partial structure factor contributions for $\mathrm{NaNO}_{2}$. As for the analogous nitrates, the constituent atoms are now of approximately equal scattering power and so the overall $F(Q)$ is a subtle superposition of significant contributions from each partial. The figure also shows the functions as $Q(F(Q)-1)$ to highlight the high $-Q$ oscillations. Figure $4(\mathrm{c})$ shows the comparison of the simulated and experimental total scattering functions for $\mathrm{KNO}_{2}$ and the breakdown at $T=800 \mathrm{~K}$ into the six partial structure factors. The agreement between simulation and experiment is probably the poorest here.

\subsection{Nitrates and nitrites. Q-space temperature dependence.}

Figure 5(a) shows the simulated total scattering functions as a function of temperature for $\mathrm{LiNO}_{3}$ along with the experimentally determined function. The first peak (at $Q \sim 2 \AA^{-1}$ ) shifts to lower $Q$ as $T$ increases in line with the decrease in density. The small feature highlighted at $Q \sim 2.8 \AA^{-1}$ appears strongly $T$-sensitive and reflects the evolution of mainly the OO partial structure factor, $S_{O O}(Q)$, whose temperature-dependence is shown in figure $5(\mathrm{~b})$. The implication is that there may be non-trivial structural changes emerging as the temperature is varied.

Figure 5(c) shows the simulated total scattering functions as a function of temperature for $\mathrm{NaNO}_{3}$ along with the experimentally determined function. The position and intensity of the first peak in $F(Q)$ changes significantly with $T$ which is attributable to strong $T$-dependent changes in the $\mathrm{NaN}$ and $\mathrm{NaNa}$ contributions (see figure $4(\mathrm{~b}))$.

Figure 5(d) shows the simulated total scattering functions as a function of temperature for $\mathrm{KNO}_{3}$ along with the experimentally determined function. As for $\mathrm{NaNO}_{3}$, the first peak (here at $Q \sim 2.1 \AA^{-1}$ ) appears most temperature sensitive.

Figures 5(e) and (f) show the simulated total scattering functions as a function of temperature for $\mathrm{NaNO}_{2}$ and $\mathrm{KNO}_{2}$ along with the experimentally determined function. The position and intensity of the first peak in $F(Q)$ in both cases changes significantly with $T$ with five of the six partial structure factors (all except the NN function) contributing significantly to this peak. For $\mathrm{NaNO}_{2}$ the most significant changes in

the NaNa function in which the first peak shifts from $Q \sim 2.20 \AA^{-1}$ at $T=600 \mathrm{~K}$ to $Q \sim 1.95 \AA^{-1}$ at $T=1000 \mathrm{~K}$.

\subsection{Nitrates and nitrites. Real space.}

Considering the inter-molecular partial radial distribution functions only, figure 6 (i.e. not the sharp peaks in either $g_{O O}(r)$ or $g_{N O}(r)$ which correspond to the intra-molecular contributions) the distributions follow the expected ordering based on the ion charges with the shortest length-scale appearing in the M-O correlations. As the cation size increases the M-O peak shifts outwards to sit over the low $-r$ tail of the $\mathrm{O}-\mathrm{O}$ term 
reflecting the increase in the $\mathrm{M}-\mathrm{O}$ coordination numbers from $<n_{M O}>\sim 5.3$ for $\mathrm{M}=\mathrm{Li}^{+}$ to $\sim 6.6$ for $\mathrm{M}=\mathrm{Na}_{4}^{+}$to $\sim 7.9$ for $\mathrm{M}=\mathrm{K}_{4}^{+}$to $\sim 9.1$ for $\mathrm{M}=\mathrm{NH}_{4}^{+}$.

Figure 7 shows the first intermolecular peak positions as a function of temperature for the three salts $(\mathrm{M}=\mathrm{Li}, \mathrm{Na}, \mathrm{K})$ for both the nitrates and nitrites. In all cases the majority of the peaks shift to higher $r$ as $T$ increases (consistent with an overall reduction in the system density). However, both the MO and MN peaks shift to lower $r$ over the same temperature range with concomitant reductions in the respective mean coordination numbers $[91,92]$.

Figure 6 shows the OO, NN and NO partial pair distribution functions for the $\mathrm{Li}, \mathrm{Na}$ and $\mathrm{K}$ nitrates and nitrites over the $T=600-1000 \mathrm{~K}$ range. In all cases the first (sharp) peaks in the NO and OO functions reflect the molecular anion geometries (and are related to the Fourier transform of the high $-Q$ oscillations shown in figure 1). The real space functions show significant changes in NO and OO ordering on the inter-molecular length-scales as temperature changes. Considering first the nitrates. As the temperature is reduced the first inter-molecular peaks in both $g_{N O}(r)$ and $g_{O O}(r)$ (as $r \sim 3.8 \AA$ and $3.1 \AA$ respectively) shift to smaller $r$. Similar behaviour is observed for the nitrites with additional changes observed in $g_{N N}(r)$ not present in the analogous nitrates. For the nitrites $g_{N N}(r)$ appears to develop a short length-scale (at $r \sim 3 \AA$ ) which strengthens as the temperature is reduced and which appears stronger for the larger cations. The pair distribution functions in the figure highlight the subtle but key structural differences between chemically-related nitrates and nitrites. These differences mean that the nitrite distributions, for example, cannot be simply obtained from the nitrate analogues by removing a single $\mathrm{O}$ atom from each $\mathrm{NO}_{3}^{-}$anion. Figure 8 shows molecular graphics "snapshots" of the sodium nitrate and nitrite (both at $T=600 \mathrm{~K}$ ). For the nitrite system "bonds" have been drawn between N-N pairs for which the separation, $r_{N N}<3.4 \AA$ (corresponding to the emerging length-scale seen in $g_{N N}(r)$ in figure 6). The presence of relatively closely approaching pairs of $\mathrm{NO}_{2}^{-}$anions is clear.

To help understand these changes and differences we calculate the cation-anion angular distribution functions, $n(\theta)$, as proposed by Yamaguchi et al [52]. These are effectively angular distribution functions which contain information regarding the distribution of the cations with respect to the planes containing the nearest-neighbour molecular anions. The preferential distribution of the alkali cations with respect to the $\mathrm{NO}_{3}^{-}$geometry has also been considered in terms of specific sites (labelled "A""D") which the cations may occupy [53]. The sites labelled "A", "B", "C", and "D" correspond to angles, $\theta$, of $40^{\circ}, 90^{\circ}, 0^{\circ}$ and $90^{\circ}$ respectively with the "B" and "D" sites lying in different locations in the plane of the anion. Figure 9 (a) shows a schematic of a single nitrate anion in order to highlight the definition of the angle $\theta$ (with respect to the vector $\mathbf{N}_{i}$ normal to the molecular plane. For the nitrates the plane is defined as that containing the three oxygen atoms whilst, for the nitrites, the only definition is that the plane contains the two oxygen and one nitrogen atom. The resultant normal vectors to these planes are correlated with vectors taken from the respective nitrogen centre to the nearest-neighbour cations (defined as sitting at distances shorter than the first minimum 
in the respective $g_{M N}(r)$ pair distribution functions). Figure $9(\mathrm{~b})$ shows $n(\theta) / \sin (\theta)$ for both the nitrates and nitrites as a function of temperature. Both sets of systems show key similarities of behaviour which are dependent upon both the temperature and the cation identity (size). For the nitrates, the function for the smallest $\left(\mathrm{Li}^{+}\right)$cation shows a peak at $\theta \sim 90^{\circ}$ at high temperature which broadens and flattens out as the temperature is reduced. Comparison of the first peak positions for the MN, MO and NO pairs shows the $\theta=90^{\circ}$ peak to correspond more to a "B" rather than a "D" site (for the latter, these peak positions would have to be additive as $\left.r_{M N}=r_{M O}+r_{N O}\right)$. Generally, however, the definition of the four types of site appears less informative than considering the full angular distributions in figure $9(\mathrm{~b})$ with the fraction of "A", "B" and "C" sites obtainable by considering the $n(\theta) / \sin (\theta)$ at $\theta=40^{\circ}, 90^{\circ}$ and $0^{\circ}$ respectively. The largest $\left(\mathrm{K}^{+}\right)$cation shows a significant peak at $\theta \sim 60^{\circ}$ at low temperature which shifts to higher angle as the temperature is increased. The $\mathrm{Na}^{+}$cation shows intermediate behaviour. Overall, the smaller cations show angular distributions which tend to those shown by the larger cations, but at lower temperature, consistent with thermal expansion.

Figure 6 indicates that the shortest NO inter-molecular length-scale is approximately constant for these systems whilst the NN nitrite functions show a significant feature at low $r(r \sim 3 \AA)$ indicative of specific short-range order facilitated by the geometry and charge distribution of the $\mathrm{NO}_{2}^{-}$anions and which is absent from the corresponding nitrate functions. To consider further the origin of this length-scale pair distribution functions are constructed weighted by the dot product of the molecular dipoles on each contributing anion site. Since the nitrite anion has a fixed formal charge

(i.e. $\sum_{i} q_{i} \neq 0$ ) we must define a fixed point in space for each molecule about which we can define the dipole, here determined with respect to the molecular centre of mass. Figure 10 shows the distribution function

$$
g_{\mu \mu}(r)=\sum_{i} \sum_{j} \boldsymbol{\mu}_{i} \boldsymbol{\mu}_{j} \delta\left(\mathbf{r}-\mathbf{r}_{i}-\mathbf{r}_{j}\right)
$$

where $\mu_{i(j)}$ is the molecular dipole on the nitrite anion labelled $i(j)$. The functions show a strong negative correlation at the short $(r \sim 3 \AA)$ length-scale indicating that the molecular dipoles on these sites tend to be pointing directly at each other. The correlation reduces as the temperature is increased for all three systems and becomes stronger as the cation size is increased. The "snapshot" of $\mathrm{NaNO}_{2}$ shown in figure 8 highlights the pairs of closely approaching $\mathrm{NO}_{2}^{-}$anions and also highlights their relative orientation.

\subsection{Behaviour of the Molecular Anion.}

Figure 11(a) shows the distribution of charges on the oxygen atoms for the three nitrate and three nitrite systems studied (all at $T=800 \mathrm{~K}$ ). The charge distributions shift to higher $q_{O}$ as the cation size decreases (with mean values of $<q_{O}>=-0.663 e,-0.648 e$ and $-0.633 e$ for the $\mathrm{Li}, \mathrm{Na}$ and $\mathrm{K}$ nitrates and $\left\langle q_{O}>=-0.795 e,-0.752 e\right.$ and $-0.733 e$ for the corresponding nitrites). These changes are consistent with the greater inductive 
effect associated with the closer approach of the smaller cations. The standard deviations of the oxygen charges on each molecular anion are relatively small ( $<10 \%$ variation compared to the mean values).

In order to characterise the out of plane distortions of the $\mathrm{NO}_{3}^{-}$anion, figure $11(\mathrm{~b})$ shows the out of plane dipole moments for the $\mathrm{Li}$, $\mathrm{Na}$ and $\mathrm{K}$ nitrates as a function of temperature. Here we determine the vector normal to the plane containing the three oxygen atoms and evaluate the dipole along this direction using the molecular centre of mass as the fixed reference point. The out of plane polarization increases with temperature and decreases as the cation size increases.

\section{Conclusions.}

A series of high energy X-ray diffraction measurements have been taken on alkali nitrate, ammonium nitrate and alkali nitrite liquids which are combined with containerless techniques to better access the liquid state. The same systems have been modelled using molecular dynamics simulations with potentials developed that allow for both the flexibility of, and fluctuations of the internal charge distribution within, the molecular anions. The flexibility of the anion allows out-of-plane polarisation to develop for the planar anions. This approach allows for exploration of the influence of temperature on the liquid structure with the simulation results highlighting the emergence of a temperature-dependent length-scale. The charge distributions of the molecular anions influence the spatial distribution of cations, in particular, their spatial relationship with respect to the plane of the molecular anion. The simulation results highlight the emergence of a strong short length-scale in the nitrites and a more subtle similar effect in the nitrates. In the former case the dipoles formed by the molecular geometry effectively point towards each other forming the short length-scale.

The emergence of non-trivial structures within the nitrate and nitrite liquids is consistent with our earlier work on carbonates [17]. In contrast to the carbonates, however, there is no evidence to indicate formation of chains. In the real space analysis the emergent length scales are shown most clearly in the N-N correlations with a strong effect in the nitrites and a much weaker effect for the nitrates. The differences between the carbonate and nitrate and nitrite systems is a reflection of the charge balance on the respective molecular anions that results from the fluctuating charge model. The

evidence for the development of intermediate range order in the nitrogen-bearing systems is most apparent in the temperature-dependence of the $\mathrm{N}-\mathrm{N}$ correlations and the contrast between nitrate and nitrite structures. This can be viewed in terms of different depth minima in the energy landscape. In the case of carbonates the minima in the potential energy surface are deeper since the charge on the carbonate anion has greater magnitude, whereas for both nitrates and nitrites the charge is less and minima shallower, this means that the nitrate and nitrite correlations are more temperature-dependence and the liquids consequently more fragile.

The combined fluctuating charge/flexible anion simulations reproduce the 
experimental data and allows exploration of parameter space which may transfer readily to other molten salt systems. Overall, a goal of this work is to explore the potential development of low-dimensional complexes in sulphate, carbonate, nitrate and nitrite systems and the temperature-dependence of these structures. The differences between the carbonate and nitrate structures are attributed to differences in electronegativity and suggests that the liquids formed from mixed molecular anions may be complex. In this approach simulations of liquids, based on chemically realistic models, and compared with new diffraction data shows the development of structure on different length scales. Their temperature-dependence and influence on dynamics and self-diffusion can be developed and behaviour predicted and will be the subject of future work.

\section{Acknowledgements.}

This work was supported by the U.S. DOE Argonne National Laboratory under contract number DE-AC02-06CH11357. Diffraction measurements on recovered samples were performed at the Materials Characterisation Laboratory, ISIS facility, Rutherford Appleton Laboratory. 
[1] Nils Breidenbach, Claudia Martin, Henning Jockenhoefer, and Thomas Bauer. Thermal Energy Storage In Molten Salts: Overview Of Novel Concepts And The DLR Test Facility TESIS. In Droege, P, editor, 10th International Renewable Energy Storage Conference, IRES 2016, volume 99 of Energy Procedia, pages 120-129, 2016. 10th International Renewabie Energy Storage Conference (IRES), Dusseldorf, GERMANY, MAR 15-17, 2016.

[2] V. M. B. Nunes, C. S. Queiros, M. J. V. Lourenco, F. J. V. Santos, and C. A. Nieto de Castro. Applied Energy, 183:603-611, 2016.

[3] R. Serrano-Lopez, J. Fradera, and S. Cuesta-Lopez. Chemical engineering and processing, 73:87$102,2013$.

[4] Thomas Bauer, Nicole Pfleger, Nils Breidenbach, Markus Eck, Doerte Laing, and Stefanie Kaesche. Applied Energy, 111:1114-1119, NOV 2013.

[5] Jess C. Gehin and Jeffrey J. Powers. Liquid Fuel Molten Salt Reactors for Thorium Utilization. Nuclear Technology, 194(2, SI):152-161, 2016.

[6] D. Heuer, E. Merle-Lucotte, M. Allibert, M. Brovchenko, V. Ghetta, and P. Rubiolo. Annals of Nuclear Energy, 64:421-429, 2014.

[7] K. M. Goff, J. C. Wass, K. C. Marsden, and G. M. Teske. Nuclear Engineering and Technology, 43(4):335-342, 2011.

[8] Christian Le Brun. Journal of Nuclear Materials, 360(1):1-5, 2007.

[9] Nicholas Boerema, Graham Morrison, Robert Taylor, and Gary Rosengarten. Solar Energy, 86(9):2293-2305, 2012.

[10] Doerte Laing, Carsten Bahl, Thomas Bauer, Michael Fiss, Nils Breidenbach, and Matthias Hempel. Proceedings of the IEEE, 100(2):516-524, 2012.

[11] Doerte Laing, Thomas Bauer, Nils Breidenbach, Bernd Hachmann, and Maike Johnson. Applied Energy, 109(SI):497-504, 2013. 12th International Conference on Energy Storage Innostock, Lleida, SPAIN, MAY 16-18, 2012.

[12] Kevin Coscia, Tucker Elliott, Satish Mohapatra, Alparslan Oztekin, and Sudhakar Neti. Journal of Solar Energy Engineering-Transactions of the ASME, 135(2), 2013.

[13] Joseph G. Cordaro, Nicholas C. Rubin, and Robert W. Bradshaw. Journal of Solar Energy Engineering-Transactions of the ASME, 133(1), 2011.

[14] G.J.Janz. J. Phys. Chem. Ref. Data, 17:1, 1988.

[15] G.J.Janz, U. Krebs, H. F. Siegenthaler, and R. P. T. Tomkins. J. Phys. Chem. Ref. Data, 1:1, 1972.

[16] D.Corradini, P.A.Madden, and M.Salanne. Faraday Discuss., 190:471, 2016.

[17] M. C. Wilding, M. Wilson, O. L. G. Alderman, C. Benmore, J. K. R. Weber, J. B. Parise, A. Tamalonis, and L. Skinner. Sci. Rep., 6:24415, 2016.

[18] Anne-Laure Rollet and Mathieu Salanne. Studies of the local structures of molten metal halides. In Webb, GA, editor, Annual Reports on the Progress of Chemistry 2011, Vol 107,Section CPhysical Chemistry, volume 107 of Annual Reports on the Progress of Chemistry Section CPhysical Chemistry, pages 88-123. 2011.

[19] SA Kirillov. Journal of Molecular Liquids, 76(1-2):35-95, 1998.

[20] G.Voehringer and J.Richter. Z. Naturforsch, 56a:337, 2001.

[21] A.K.Adya, R.Takagi, K.Kawamura, and M.Mikami. Mol. Phys., 62:227, 1987.

[22] A.K.Adya and G.W.Neilson. Mol. Phys., 69:747, 1990.

[23] A.K.Adya and G.W.Neilson. Mol. Phys., 71:1091, 1990.

[24] A.K.Adya, G.W.Neilson, I.Okada, and S.Okazaki. Mol. Phys., 79:1327, 1993.

[25] C. A. Angell. Science, 267(5206):1924-1935, 1995.

[26] G.J.Janz, F. W. Dampier, G. R. Lakshminarayanan, P. K. Lorenz, and R. P. T. Tomkins. National Bureau of Standards Reference Data Series, 15:1, 1966.

[27] J K R Weber and P C Nordine. Microgravity Science And Technology, 7(4):272-282, 1995.

[28] J K R Weber. European Journal Of Solid State And Inorganic Chemistry, 34(7-8):847-859, 1997.

[29] J K R Weber, C J Benmore, Q Mei, and M C Wilding. In-situ x-ray structure measurements on 
aerodynamically levitated high temperature alumina. In Synchrotron Radiation in Materials Science, volume 1092 of AIP Conference Proceedings, pages 79-83. 2009.

[30] J K R Weber, C J Benmore, L B Skinner, J Neuefeind, S K Tumber, G Jennings, L J Santodonato, J Din, J Du, and J B Parise. Journal of Non-crystalline Solids, 383:49-51, 2014.

[31] J. K. R. Weber, A. Tamalonis, C. J. Benmore, O. L. G. Alderman, S. Sendelbach, A. Hebden, and M. A. Williamson. Review of Scientific Instruments, 87(7), 2016.

[32] J. K. R. Weber, J. E. Rix, K. J. Hiera, J. A. Tangeman, C. J. Benmore, R. T. Hart, J. E. Siewenie, and L. J. Santodonato. Physics And Chemistry Of Glasses, 46(4):487-491, 2005.

[33] F. Demmel, L. Hennet, S. Brassamin, D. R. Neuville, J. Kozaily, and M. M. Koza. Physical Review $B, 94(1), 2016$.

[34] N. Yodoshi, R. Yamada, A. Kawasaki, and A. Makino. Journal of Alloys and Compounds, 643(1):S2-S7, 2015.

[35] O. L. G. Alderman, L. B. Skinner, C. J. Benmore, A. Tamalonis, and J. K. R. Weber. Physical Review B, 90(9), 2014.

[36] L. B. Skinner, C. J. Benmore, J. K. R. Weber, M. A. Williamson, A. Tamalonis, A. Hebden, T. Wiencek, O. L. G. Alderman, M. Guthrie, L. Leibowitz, and J. B. Parise. Science, 346(6212):984-987, 2014.

[37] L. B. Skinner. Science, 349(6245, SI):247, 2015.

[38] A. K. Soper. Phys. Rev. B, 72:104204, 2005.

[39] PA Madden and M Wilson. Chemical Society Reviews, 25(5):339-\&, 1996.

[40] M.Wilson. Phys. Chem. Chem. Phys., 14:12701, 2012.

[41] N.C.Pyper. Adv. Solid State Chem., 2:223, 1991.

[42] A.K.Rappe and W.A.Goddard III. J. Phys. Chem., 95:3358, 1991.

[43] Y-P.Liu, K.Kim, B.J.Berne, R.A.Friesner, and S.W.Rick. J. Chem. Phys., 108:4739, 1998.

[44] M.C.C. Ribeiro and L.C.J. Almeida. J. Chem. Phys., 110:11445, 1999.

[45] S.W.Rick, S.J.Stuart, and B.J.Berne. J. Chem. Phys., 101:6141, 1994.

[46] M.C.C. Ribeiro and L.C.J. Almeida. J. Chem. Phys., 113:4722, 2000.

[47] M.C.C Ribeiro. J. Chem. Phys., 117:266, 2002.

[48] M.C.C. Ribeiro. Phys. Rev. B, 63:094205, 2001.

[49] M.C.C. Ribeiro. Phys. Rev. B, 61:3297, 2000.

[50] S.M.Urahata and M.C.C.Ribeiro. PCCP, 5:2619, 2003.

[51] J Zarzycki. Discuss. Faraday Soc., 32:38, 1961.

[52] T.Yamaguchi, I.Okada, H.Ohtaki, M.Mikami, and K.Kawamura. Mol. Phys., 58:349, 1986.

[53] H.Ohno and K.Furukawa. J. Chem. Soc. Faraday Trans. I, 74:297, 1977.

[54] K.Suzuki and Y.Fukushima. Z. Naturforsch, 32a:1438, 1977.

[55] F.A.Cotton, G.Wilkinson, C.A.Murillo, and M.Bochmann. Advanced Inorganic Chemistry. WileyBlackwell, 1999.

[56] O. L. G. Alderman, Benmore C.J., Tamalonis A., Sendelbach S., Heald S.M., and Weber R. Journal of Physical Chemistry C, 120:26974, 2016.

[57] J K R Weber, S Krishnan, C D Anderson, and P C Nordine. Spectral absorption coefficient of molten aluminum oxide from 0.385 to $0.780 \mu \mathrm{m}$. J. Am. Ceram. Soc., 78(3):583-587, 1995.

[58] Royal Society of Chemistry. www.rsc.org, 2017.

[59] G.B.Mandis, G.M.Nazin, Yu.I.Rubtsov, and V.A.Strunin. Thermal Decomposition and Combustion of Explosives and Propellants. Taylor and Francis, 2003.

[60] Y. Ivanyushenkov, K. Harkay, M. Abliz, L. Boon, M. Borland, D. Capatina, J. Collins, G. Decker, R. Dejus, J. Dooling, C. Doose, L. Emery, J. Fuerst, J. Gagliano, Q. Hasse, M. Jaski, M. Kasa, S. H. Kim, R. Kustom, J. C. Lang, J. Liu, E. Moog, D. Robinson, V. Sajaev, K. Schroeder, N. Sereno, Y. Shiroyanagi, D. Skiadopoulos, M. Smith, X. Sun, E. Trakhtenberg, I. Vasserman, A. Vella, A. Xiao, J. Xu, A. Zholents, E. Gluskin, V. Lev, N. Mezentsev, V. Syrovatin, V. Tsukanov, A. Makarov, J. Pfotenhauer, and D. Potratz. Physical Review Special TopicsAccelerators and Beams, 18(4), 2015. 
[61] Y. Ivanyushenkov, C. Doose, J. Fuerst, Q. Hasse, M. Kasa, and Y. Shiroyanagi. IEEE Transactions on Applied Superconductivity, 24(3), 2014.

[62] A P Hammersley, S O Svensson, M Hanfland, A N Fitch, and D Haeusermann. High Pressure Research, 14:235, 1996.

[63] X Y Qiu, J W Thompson, and S J L Billinge. Journal of Applied Crystallography, 37:678, 2004.

[64] Lawrie B. Skinner, Chris J. Benmore, and John B. Parise. Nuclear Instruments 83 Methods in Physics Research Section A-Accelerators Spectrometers Detectors and Associated Equipment, 662(1):61-70, 2012.

[65] T.Kato, K.Machida, M.Oobatake, and S.Hayashi. J. Chem. Phys., 89:3211, 1988.

[66] T.Kato, K.Machida, M.Oobatake, and S.Hayashi. J. Chem. Phys., 89:7471, 1988.

[67] T.Kato, K.Machida, M.Oobatake, and S.Hayashi. J. Chem. Phys., 92:5506, 1990.

[68] T.Kato, K.Machida, M.Oobatake, and S.Hayashi. J. Chem. Phys., 99:3966, 1993.

[69] G.F.Signorini, J-L.Barrat, and M.L.Klein. J. Chem. Phys., 92:1294, 1990.

[70] C.Ebner, R.Sansone, S.Hengrasmee, and M.Probst. Int. J. Quantum Chem., 75:805, 1999.

[71] J.F.Wyatt, I.H.Hillier, V.R.Saunders, J.A.Connor, and M.Barber. J. Chem. Phys., 54:5311, 1971.

[72] R.M.Lynden-Bell, M.Ferrario, I.R.McDonald, and E.Salje. J. Phys.: Condens. Matter, 1:6523, 1989.

[73] A.Pullman and H.Berthod. Chem. Phys. Lett., 81:195, 1981.

[74] J.D.Goddard, M.L.Klein, and Y.Ozaki. J. Phys. Soc. Jap., 52:1168, 1983.

[75] M.Okuda, S.Ohba, Y.Saito, T.Ito, and I.Shibuya. Acta. Cryst. B, 46:343, 1990.

[76] J.D.Axe. Phys. Rev., 167:573, 1968.

[77] W-G.Yin, C-G.Duan, W.N.Mei, J.Liu, R.W.Smith, and J.R.Hardy. Phys. Rev. B, 68:174106, 2003.

[78] D.M.Hirst and J.W.Linnett. J. Chem. Phys., 43:S74, 1965.

[79] P.Geerlings, F.De Proft, and W.Langenaeker. Chem. Rev., 103:1793, 2003.

[80] S. Nosé. J. Chem. Phys., 81:511, 1984.

[81] W. G. Hoover. Phys. Rev. A, 31:1695, 1985.

[82] D. H. Booth and V. C. Vinyard. Journal of Applied Chemistry, 1967.

[83] R Car and M Parrinello. Phys. Rev. Lett., 55(22):2471 - 2474, 1985.

[84] D C Parfitt, D A Keen, S Hull, W A Crichton, M Mezouar, M Wilson, and P A Madden. Phys. Rev. B, 72:054121, 2005.

[85] D T Cromer and J T Waber. International tables for X-ray Crystallography. Kynoch Press, Birmingham, 1974.

[86] J H Hubbell, W J Veigele, E A Briggs, R T Brown, D T Cromer, and R J Howerton. Journal of physical Chemistry Reference Data, 4(3):471, 1975.

[87] Q.Mou, C.J.Benmore, and J.L.Yarger. J. Appl. Cryst., 48:950, 2015.

[88] RD Shannon. Acta Crystallographica Section A, 32(Sep1):751-767, 1976.

[89] RD Shannon and CT Prewitt. Acta Crystallographica Section B -Structural Crystallography and Crystal Chemistry, B 25(5):925-\&, 1969.

[90] V Sidey. Acta. Cryst. B, 72:626-633, 2016.

[91] L. B. Skinner, C. J. Benmore, J. K. R. Weber, J. Du, J. Neuefeind, S. K. Tumber, and J. B. Parise. Physical Review Letters, 112(15), 2014.

[92] M. Bauchy and M. Micoulaut. EPL, 104(5), 2013. 


\begin{tabular}{cccc}
\hline Compound & $\mathrm{T} / \mathrm{K}$ & $\mathrm{T}_{m} / \mathrm{K}$ & $\mathrm{T}_{\text {decomp }} / \mathrm{K}$ \\
\hline $\mathrm{LiNO}_{3}$ & $680 \pm 8$ & $526^{*}$ & $747^{*}$ \\
$\mathrm{NaNO}_{3}$ & $619 \pm 27$ & $581^{*}$ & $802^{*}$ \\
$\mathrm{KNO}_{3}$ & $660 \pm 2$ & $610^{*}$ & $806^{*},>673^{\dagger}$ \\
$\mathrm{NH}_{4} \mathrm{NO}_{3}$ & $543 \pm 10$ & $443^{\dagger}$ & $473-533^{\dagger}$ \\
$\mathrm{NaNO}_{2}$ & $697 \pm 8$ & $544^{\dagger}$ & $>593^{\dagger}$ \\
$\mathrm{KNO}_{2}$ & $718 \pm 2$ & $714^{\dagger}$ & $>623^{\dagger}$ \\
\hline
\end{tabular}

Table 1. Sample temperatures (second column) obtained as described in the text. The third and fourth columns list the respective melting temperatures, $T_{m}$, and decomposition temperatures, $T_{\text {decomp }}$, respectively. These are taken from references * [59] and ${ }^{\dagger}[58]$ as indicated. 

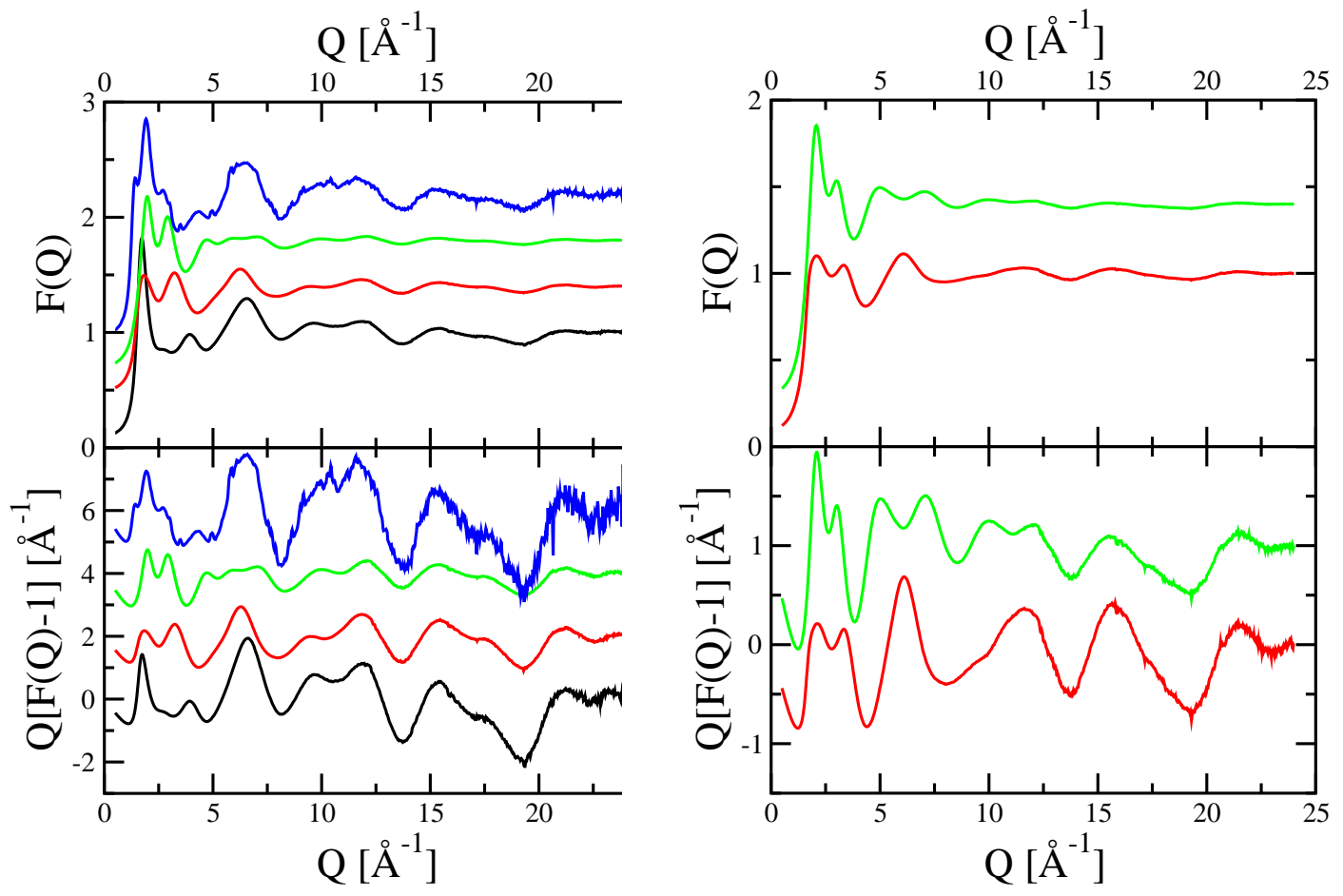

Figure 1. Total scattering functions, $F(Q)$, obtained from HEXRD for (left panels) four pure nitrate and (right panels) two pure nitrite systems. Key: black - $\mathrm{Li}^{+}$, red - $\mathrm{Na}^{+}$, green $-\mathrm{K}^{+}$, blue $-\mathrm{NH}_{4}^{+}$. The upper panels show the raw $F(Q)$ data whilst the lower panels show $Q[F(Q)-1)$ to highlight the high $-Q$ oscillations. In all panels successive systems are offset along the abscissa for clarity. 

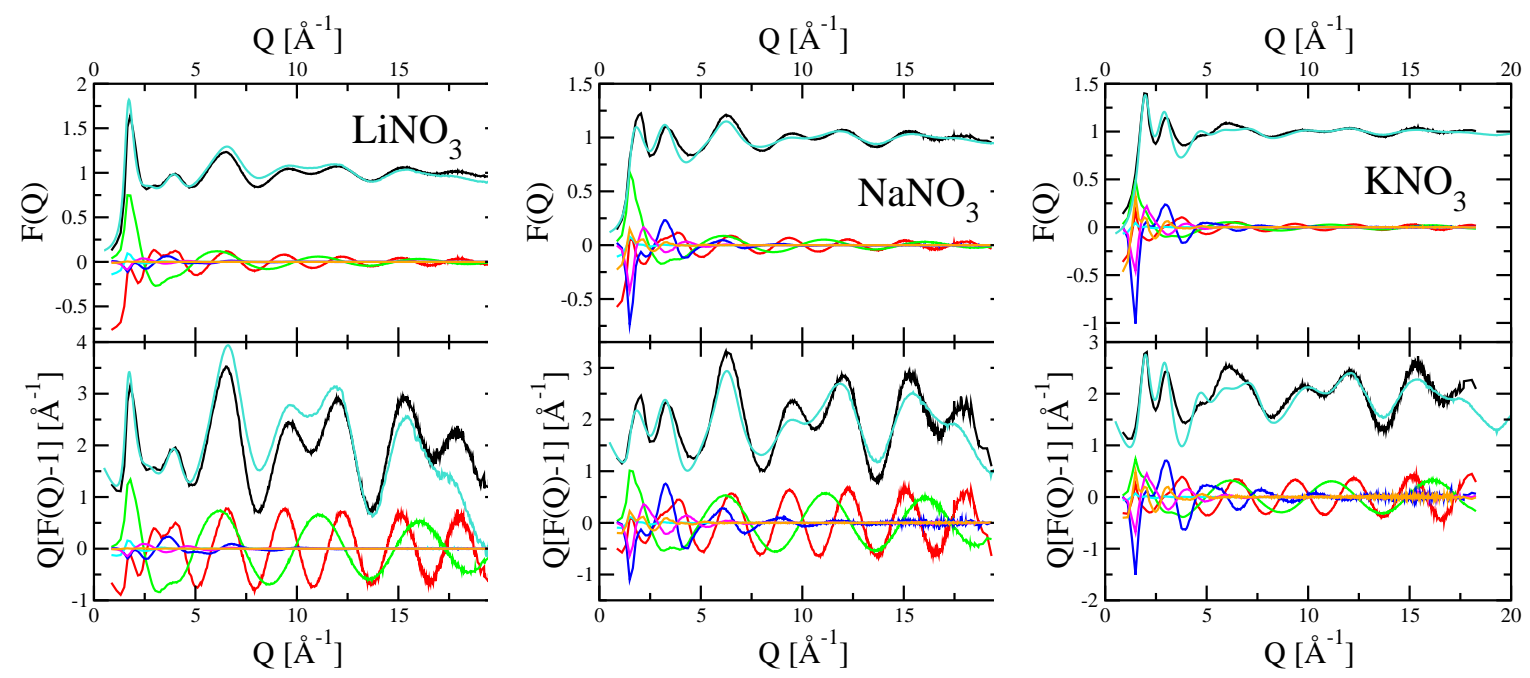

Figure 2. The total scattering functions obtained for the three pure nitrate systems from simulation with the breakdown into (X-ray weighted) partial structure factors. Left panel - $\mathrm{LiNO}_{3}$, central panel - $\mathrm{NaNO}_{3}$, right panel - $\mathrm{KNO}_{3}$. In all cases the upper panels show the data whilst the lower panels show $Q[F(Q)-1)$ or $Q\left[S_{\alpha \beta}(Q)-1\right]$ to highlight the high $-Q$ oscillations. In all cases the HEXRD patterns are shown as the turquoise lines. Key: black - $F(Q)$, red - $S_{O O}(Q)$, green - $S_{N O}(Q)$, blue $S_{M O}(Q)$, cyan - $S_{N N}(Q)$, magenta - $S_{M N}(Q)$, orange - $S_{M M}(Q)$. In all panels the total scattering functions are offset along the abscissa for clarity. 


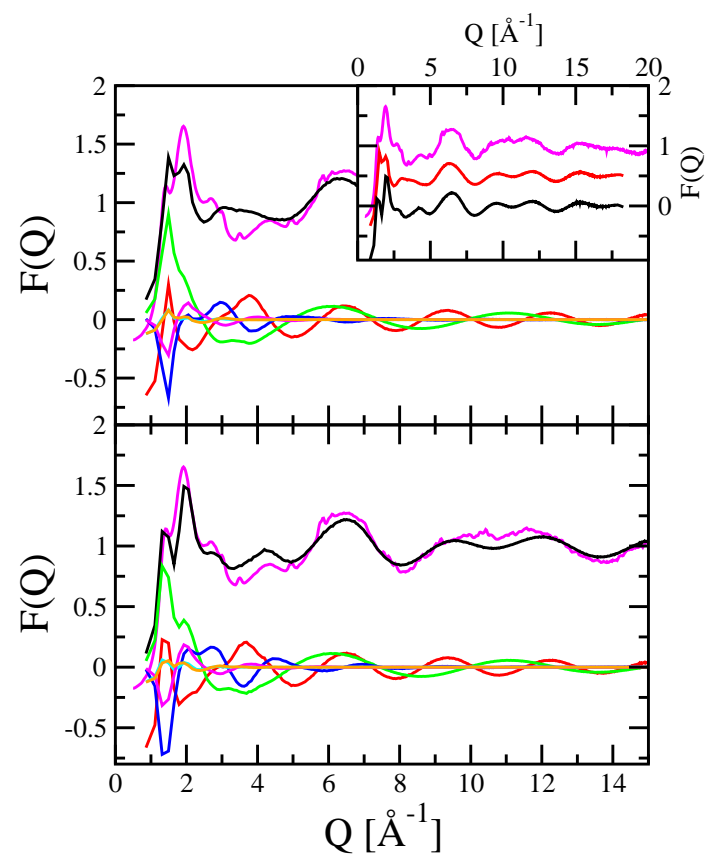

Figure 3. The main panels show the total scattering function obtained for $\mathrm{NH}_{4} \mathrm{NO}_{3}$ $F(Q)$ from simulation (black lines) and HEXRD (magenta lines) and the associated breakdowns into weighted partial structure factors (key as for figure 2. The upper panel shows the functions obtained from a model assuming the ammonium ion to have the same size as the potassium ion, whilst the lower shows the effect of increasing the cation size as discussed in the text. In both panels the total scattering functions are offset along the abscissa for clarity. The inset in the upper panel shows the total scattering functions obtained from HEXRD and from simulation for the two cation sizes. Successive curves are offset along the abscissa for clarity. 

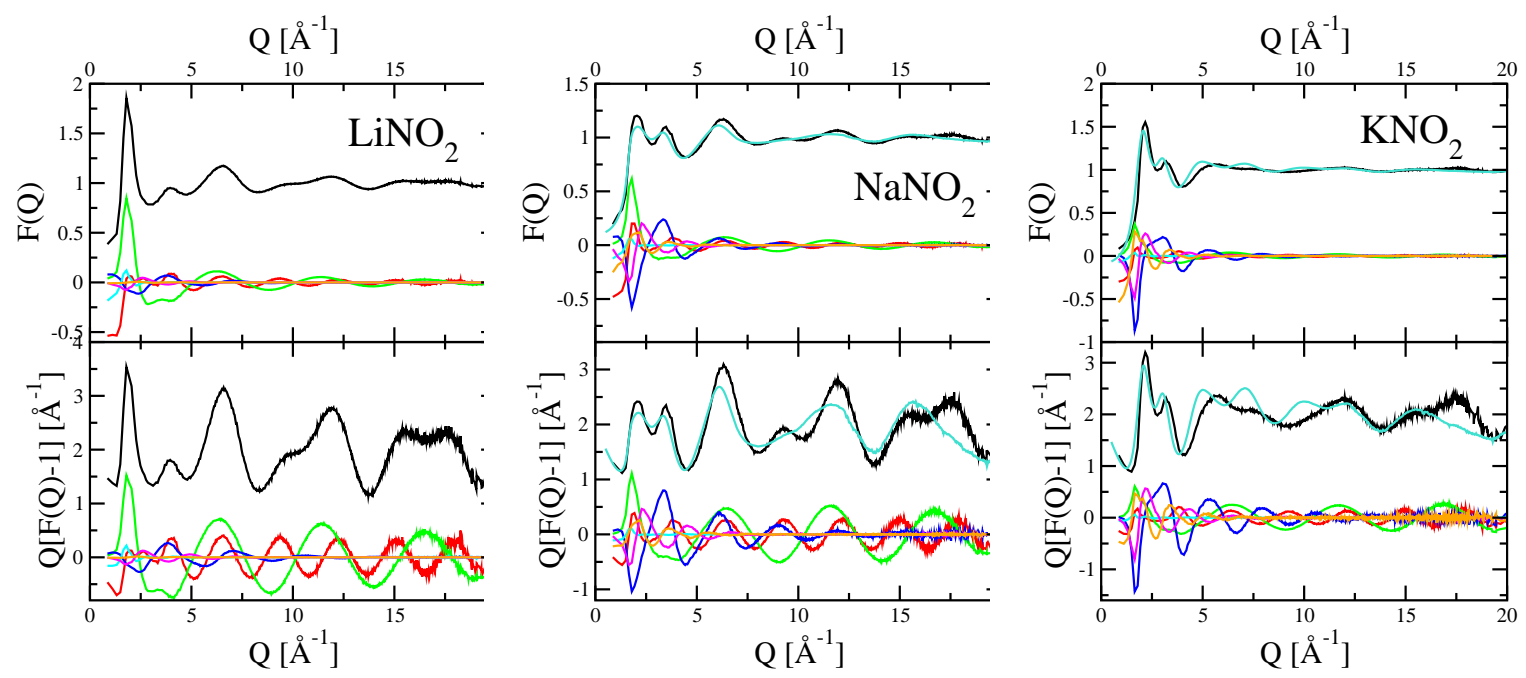

Figure 4. The total scattering functions obtained for the three pure nitrite systems from simulation with the breakdown into (X-ray weighted) partial structure factors. Left panel - $\mathrm{LiNO}_{2}$, central panel - $\mathrm{NaNO}_{2}$, right panel - $\mathrm{KNO}_{2}$. In all cases the upper panels show the data whilst the lower panels show $Q[F(Q)-1)$ or $Q\left[S_{\alpha \beta}(Q)-1\right]$ to highlight the high $-Q$ oscillations. For the sodium and potassium systems the HEXRD patterns are shown as the turquoise lines. Key: black - $F(Q)$, red - $S_{O O}(Q)$, green $S_{N O}(Q)$, blue - $S_{M O}(Q)$, cyan - $S_{N N}(Q)$, magenta - $S_{M N}(Q)$. orange - $S_{M M}(Q)$. In all panels the total scattering functions are offset along the abscissa for clarity. 

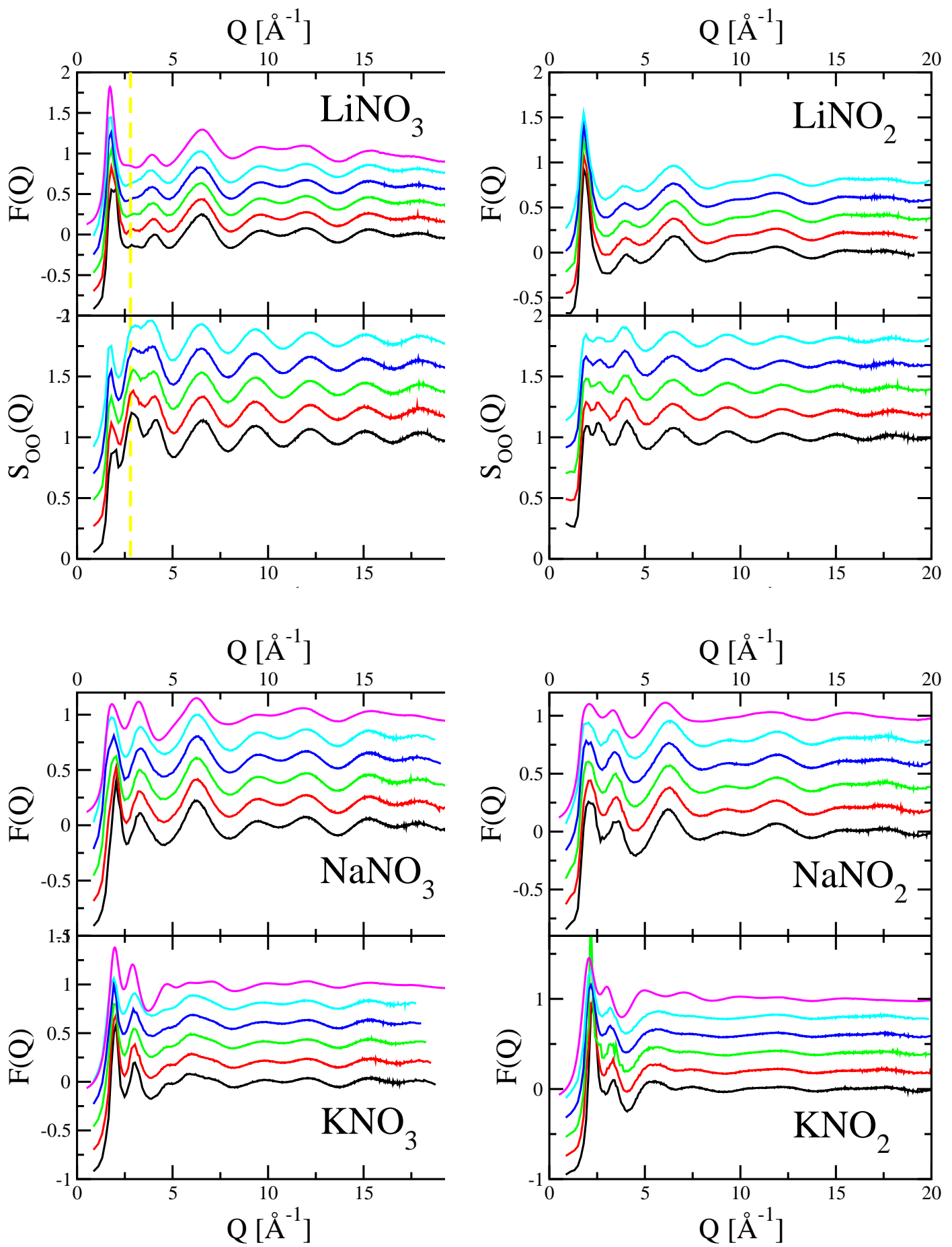

Figure 5. The total scattering functions obtained for the three pure nitrate and three pure nitrite systems from HEXRD and from simulation as a function of temperature. Key: upper panel - $\mathrm{LiNO}_{3}$, upper right panel - $\mathrm{LiNO}_{2}$, lower left panel - $\mathrm{NaNO}_{3}$ and $\mathrm{KNO}_{3}$, lower right panel $-\mathrm{NaNO}_{2}$ and $\mathrm{KNO}_{2}$. In both the upper panels the temperature evolution of the partial structure factors $S_{O O}(Q)$ are shown. In all panels the total scattering functions are offset along the abscissa for clarity with successive curves (bottom to top) showing data obtained at $T=600 \mathrm{~K}, 700 \mathrm{~K}, 800 \mathrm{~K}, 900 \mathrm{~K}$ and $1000 \mathrm{~K}$ respectively. In the five panels showing the total scattering functions the magenta lines show the HEXRD data. 

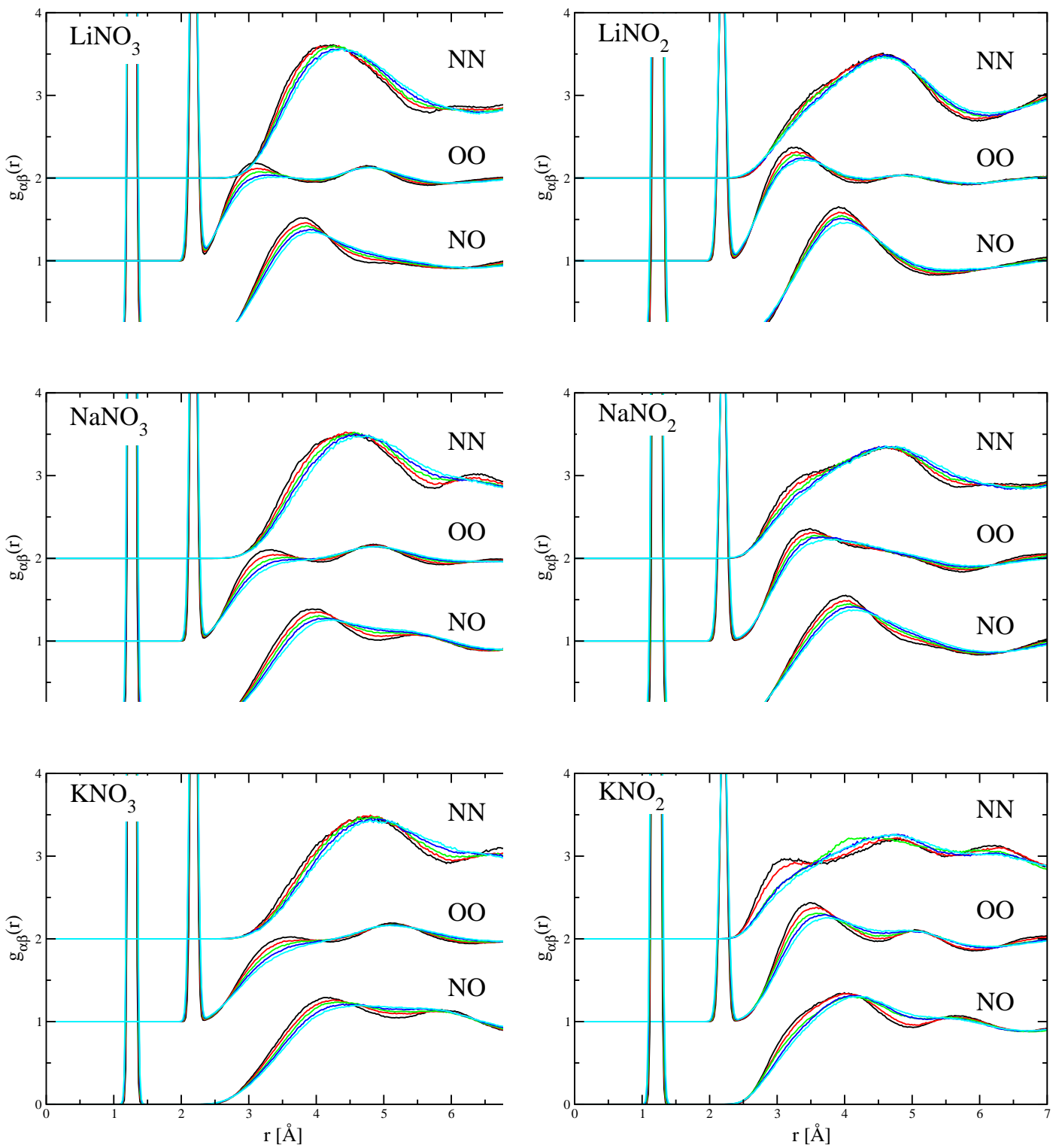

Figure 6. The evolution of the partial radial distribution functions as a function of temperature. In all panels the upper curves show $g_{N N}(r)$, the central curves $g_{O O}(r)$ and the lower curves $g_{N O}(r)$. The black-red-green-blue-cyan curves all all cases show these functions for $T=600 \mathrm{~K}, 700 \mathrm{~K}, 800 \mathrm{~K}, 900 \mathrm{~K}$ and $1000 \mathrm{~K}$ respectively. In all panels the $g_{N N}(r)$ and $g_{O O}(r)$ are offset along the abscissa for clarity. The left panels show (from upper to lower) the functions for $\mathrm{LiNO}_{3}, \mathrm{NaNO}_{3}$ and $\mathrm{KNO}_{3}$ respectively whilst the right panels show the corresponding nitrites. 

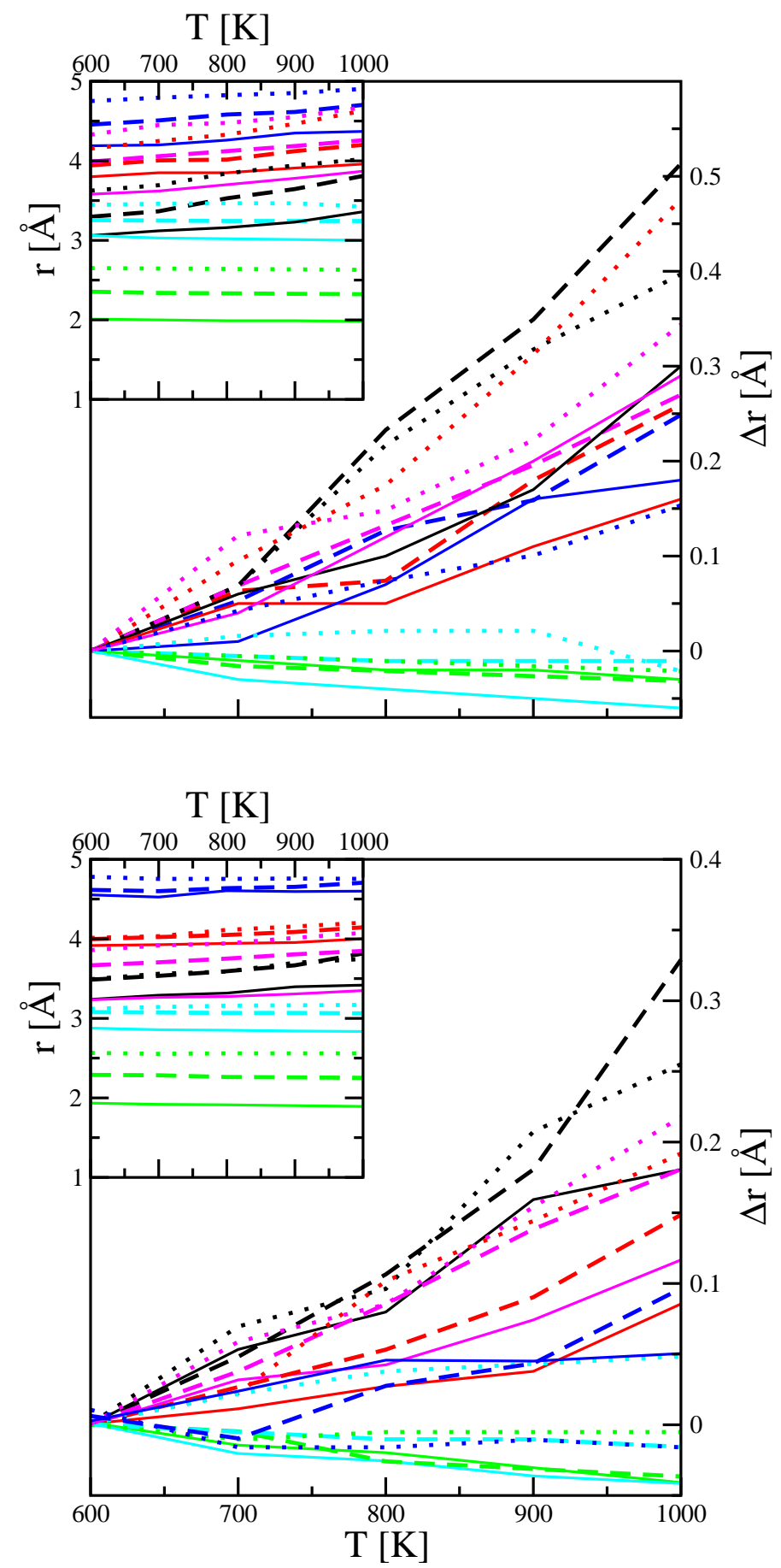

Figure 7. Temperature dependence of the first peak positions in the intermolecular partial radial distribution functions for the nitrates (left) and nitrites (right). The solid, dashed and dotted lines refer to the lithium, sodium and potassium salts respectively. Key: black - OO, red - NO, green - MO, blue - NN, cyan - MN, magenta - MM. For each case the left panel shows the absolute values the the right panel the change in value with respect to the $T=600 \mathrm{~K}$ values. 

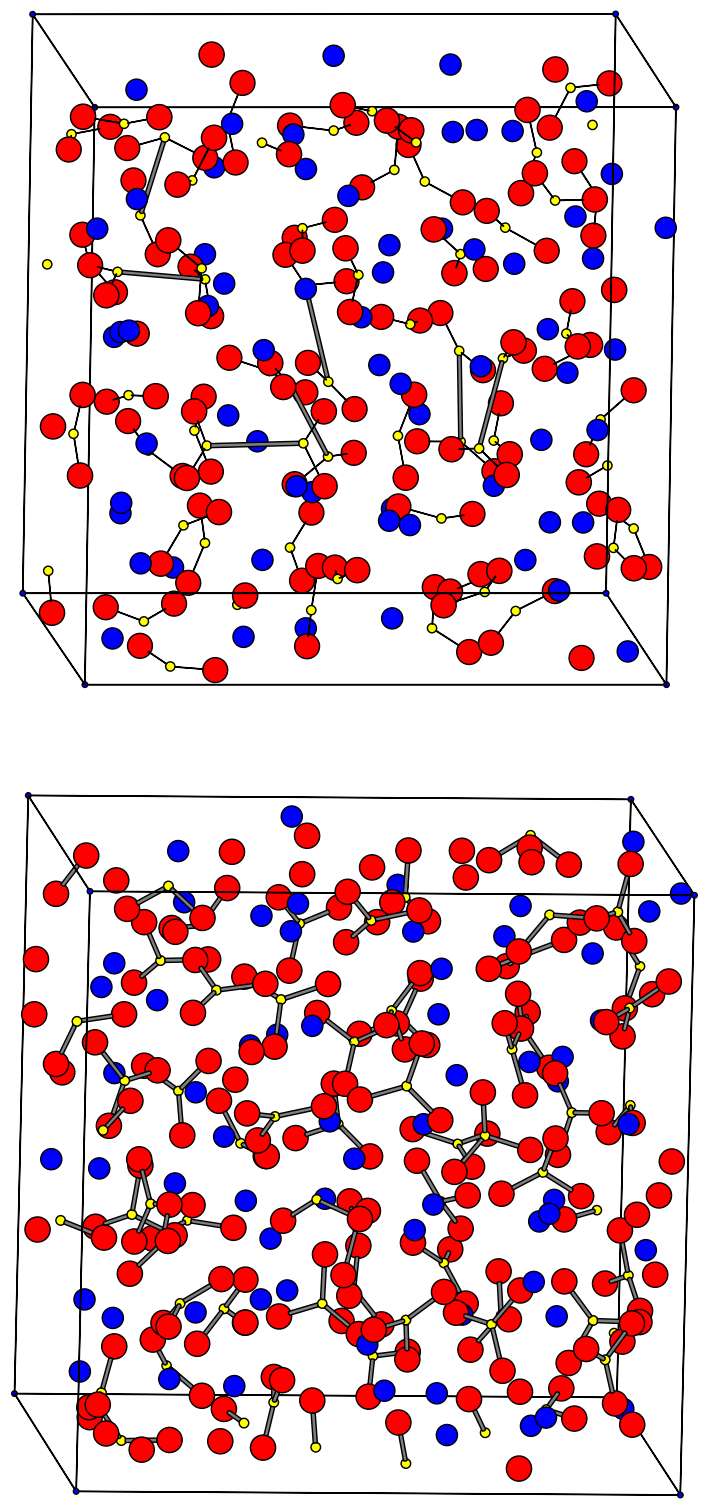

Figure 8. Molecular graphics "snapshots" of (bottom) $\mathrm{NaNO}_{3}$ and (top) $\mathrm{NaNO}_{2}$ both at $T=600 \mathrm{~K}$. In both panels the $\mathrm{N}, \mathrm{O}$ and $\mathrm{Na}$ atoms are shown as magenta, red and blue circles respectively whilst the nearest-neighbour $\mathrm{N}-\mathrm{O}$ pairs are joined by lines. In the upper panel N-N atom pairs are joined by a thick line if separated by $<3.4 \AA$ to highlight the emergence of the short length-scale (see figure 6). 

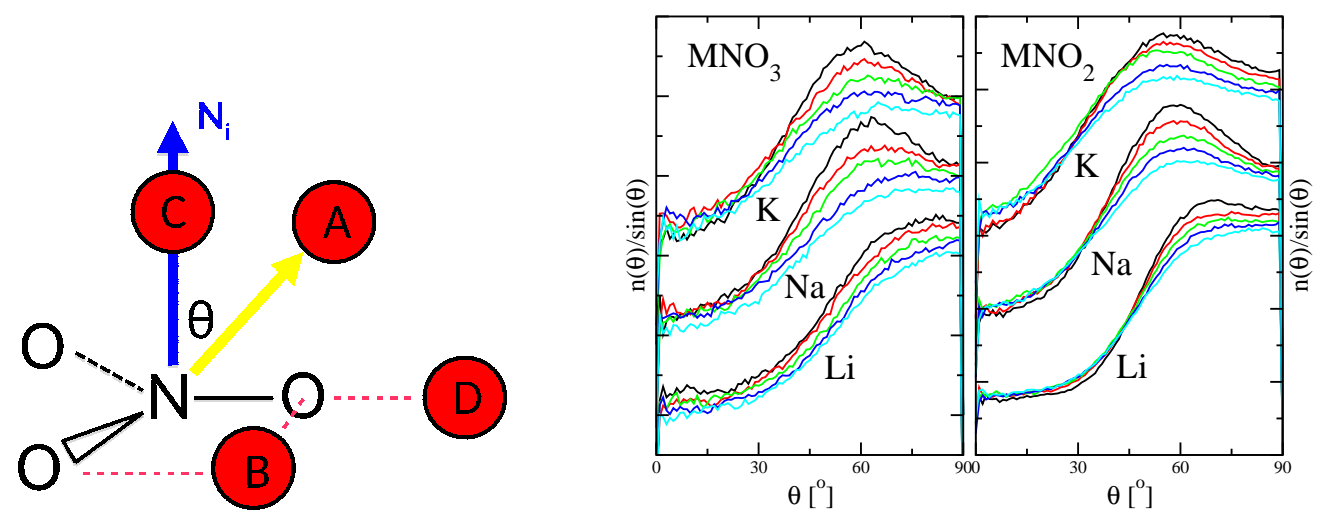

Figure 9. (Left panel) A schematic of a single nitrate anion to highlight the definition of the angle $\theta$ and the locations of the four "site types" labelled "A"- "D" (defined in [53]). (Right panel) The evolution of the angular distribution functions, $n(\theta)$, as a function of temperature for the three nitrates (left panel) and nitrites (right panel). The functions are shown (from bottom to top) as lithium salts, sodium salts and potassium salts. In both cases the black-red-green-blue-cyan shows the effect of increasing temperature.

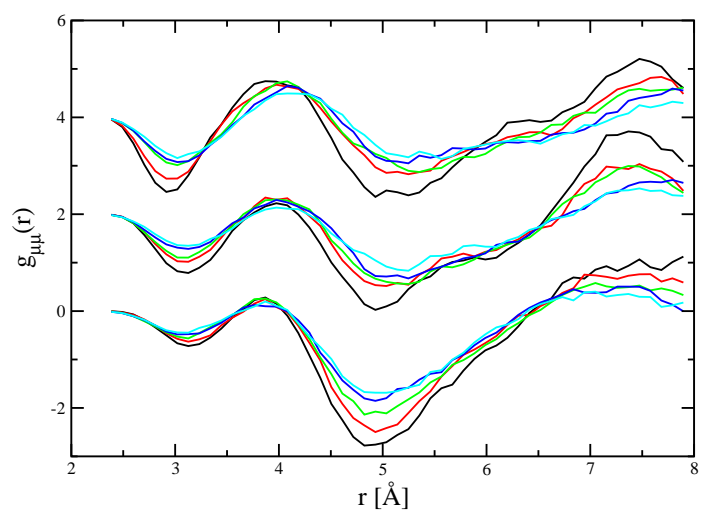

Figure 10. Radial distribution functions weighted by the molecular dipoles for the three nitrites as a function of temperature. The functions are shown (from bottom to top) in the order lithium salts, sodium salts and potassium salts. In all cases the black-red-green-blue-cyan shows the effect of increasing temperature. 

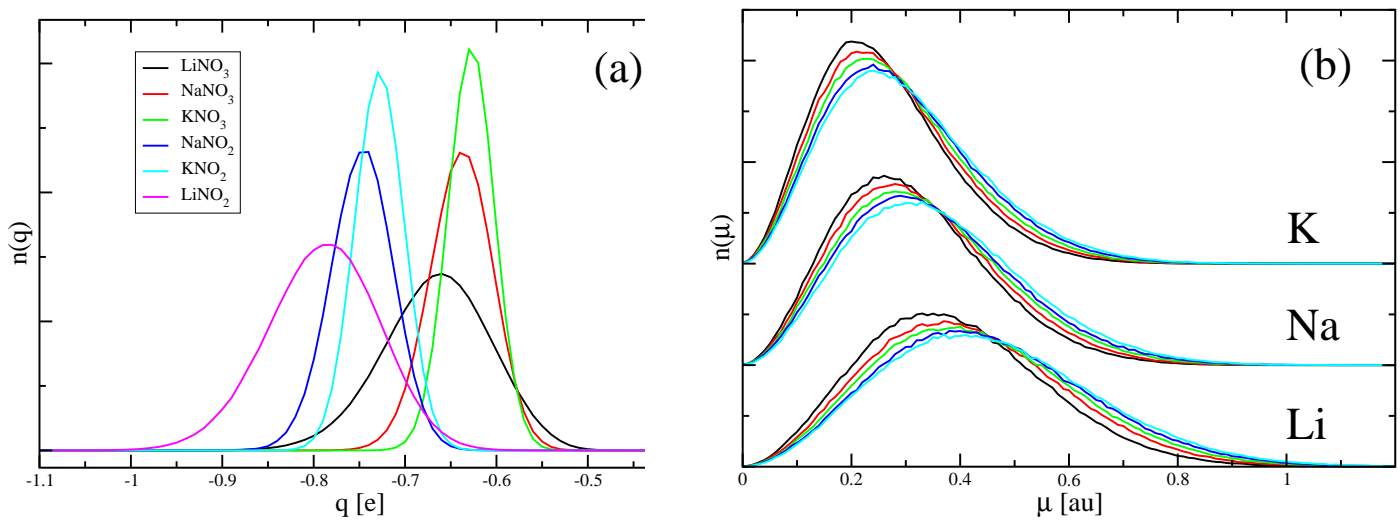

Figure 11. (a) Oxygen charge distributions for our FCM for 6 systems. (b) Out of plane dipole distributions for our FCM for 3 nitrate systems. The ordinate axis scale is arbitrary. In all cases the black-red-green-blue-cyan shows the effect of increasing temperature (from $600 \mathrm{~K}-700 \mathrm{~K}-800 \mathrm{~K}-900 \mathrm{~K}-1000 \mathrm{~K}$ ). In both (a) and (b) the ordinate scales are arbitrary. 